\title{
Decentralized Voltage Regulation in radial Medium Voltage Networks with high presence of Distributed Generation
}

\author{
Konstantinos Kotsalos ${ }^{1}$ \\ ${ }_{1}^{1}$ Department of Electrical \& Computer Engineering, Faculty of Engineering University of \\ Porto (up201609040@fe.up.pt), Efacec Energia S.A. (konstantinos.kotsalos@efacec.com)
}

\begin{abstract}
The continuous growth of distributed generation along the medium voltage distribution networks (MVDN) induces multifaceted technical challenges that have to be addressed by alternative control architecture schemes beyond the centralized strategies and the fit and forget doctrine. The active participation of DG essentially drives the network to pave towards the Smart Grid concept. This paper entails a decentralized control strategy which is based on a sensitivity analysis to stipulate the proper dispatch set-points for the DGs; hence, all nodal voltages are in permissible bounds. An overview of sensitivity approaches is presented and discussed for their adequacy to be used in MVDN. The proposed method is simulated on a 13- Node Test Feeder IEEE benchmark, while its consistency is compared with a centralized scheme.
\end{abstract}

Subject Headings. Electrical Engineering, Electrical Energy

Author Keywords. Voltage Regulation, Medium Voltage Network, Distribution Network, Decentralized control, sensitivity analysis

\section{Introduction}

The emphasis on climate change policy across EU has been to cut greenhouse gas emissions through reductions and efficiency savings in the power sector. There is a clear focus on promoting low carbon and renewable energy technologies for generation with the new and binding targets of $20 \%$ by 2020 . The last decades this policy commitment has been matched with the national schemes promoting remarkable integration of energy share from renewable sources. The main drivers are global awareness of environmental pollution as well as the significant global energy demand growth lead to further propel the integration of Distributed Generation (DG) and Distributed Energy Resources (DER) into power system.

The ever-increasing presence of DERs takes place predominantly in Distribution Networks (DN) (i.e. Medium and Low Voltage Network) possibly leading to technical challenges. The integration of DGs is progressively facing limits intrinsic to current power systems, which have a top down structure based on centrally generated power. The continuous growth of DERs integration pose challenges to current DN operation since they distort basic assumptions in distribution network planning [Reponen et al. 2016]. The electric power system is evolving from large central generating stations interconnected with customers through grids of transmission and distribution lines into a system that includes substantial DER. Traditionally, the power system was designed to accept solely bulk power from the transmission network to the consumers at lower voltages. These networks were designed through deterministic studies considering critical cases so that distribution networks could operate with the minimum amount of controls [Pecas Lopes et al. 2007]. This refers to the fit and forget dogma, 
which was additionally adopted for the interconnection of DERs (i.e. usually called connect and forget) meaning that DERs did not actively participate in the operation and planning of the power systems. The connection of DERs along the DN creates bidirectional power flow, with the possibility of occurring constraints such as branch congestion and voltage unbalances among downstream feeders.

In order to technically welcome further integration of DG units, multiple grid reinforcements may need to be planned. Nevertheless, the latter could be surpassed if a smoothly integration of these decentralized sources into the electricity system is promoted. This could be substantially addressed by evolving networks with more intelligence, paving the way of the so-called smart grids. More than a significant technical challenge, it is an evolutionary opportunity to progress towards the Smart grid (SG) concept integrating smart metering, advanced Information and Communication Infrastructures (ICT), Intelligent Electronic Devices (IED) and smart inverters in addition to distribution automation to ensure a proper interface between DGs and the electric utilities. This welcomes the transit to the Active Network Management (ANM) by utilizing the breakthroughs occurring within the framework of SG. One of the key drivers of ANM is the flexible management of Active and Reactive Power of DERs; thus, this properly contributes to reassure the distribution's network operation.

The voltage in Medium Voltage Distribution Networks (MVDN) is currently regulated by the On-Load-Tap-Changer of the primary transformer (i.e. HV/MV transformer) as well as with the installed shunt capacitors and inductances or even more efficiently with the use of Dynamic Voltage Restorer (DVR) and intermediate SVRs (Shunt Voltage Regulators). Nevertheless, the aforementioned techniques of regulating voltage are not capable to provide proper regulation to DN with high presence of DG. In [Farag et al. 2012 and Vovos et al. 2007] the impact of high DG penetration is presented showing analytically that the interconnection of DGs along a feeder distort the monotonic formulation of voltage profile due to the bidirectional power flows. Conversely, DGs can essentially managed and provide voltage support (i.e. ride through) during voltage sags in emergency conditions. This is feasible in favor of the smart inverters which are capable to be dynamically tuned into different set-points by the mean that the DGs can be operated with a variable power factor, injecting or absorbing reactive and active power from the network.

On this paper a decentralized voltage control strategy is proposed based a sensitivity analysis theory which indicates the most proper (i.e. most sensitive) DG unit that can be dispatched in order to restore the nodal voltage which exceeds the permissible bounds. The typical controls on the conventional structure of power system were purely centralized provided that such schemes can deliver the optimal performance point; requiring augmented telecommunication infrastructures. Nevertheless, the high DG integration into DNs induces technical challenges [Pecas Lopes et al. 2007] which reach the limit of centralized decision making schemes. Additionally, the increase of uncertainties (intermittent nature of RES, responsive loads, Storage Systems, self-healing and reconfiguration) and the endeavor to transit to "plug and play" features, imply that a level of distributed intelligence among the DN have to be facilitated.

Amid to these novel requirements for the future power network, a decentralized control algorithm is proposed which can be implemented into peer-to-peer communication based on the concept of multi-agent based system (MAS). In the literature [Nguyen et al. 2007, Jiang 2006, Lund 2007, Oyarzabal et al. 2005, Pipattanasomporn et al. 2009 and Nguyen 2008], there are some previous works made that propose distributed voltage control schemes using 
MAS, which provide the proper tuning of dispatch set-points pertaining the Reactive Power output of DGs.

The goal of this framework is to present a consistent decentralized control algorithm that is aligned to the SG concept such that can be implemented within the cutting edges taking place on the Information Communications Technology (ICT).

In section 2 an overview of different approaches of sensitivity approaches is presented while section 3 portrays the decentralized algorithm scheme. The results of the simulations are thoroughly discussed in Section 4.

\section{Sensitivity Analysis}

Observing progressive injections of active power occurring in a generator's output the voltage of the interconnected busbar tends to rise proportionally. Assuming a DG is connected to Bus g -Figure 1; any voltage in bus $s$ can be described from equation (1) where it is clearly depicted that the voltage variation due to active or reactive power variation is inevitably connected with the ratio $X / R$ the characteristic parameters of the line that intersects.

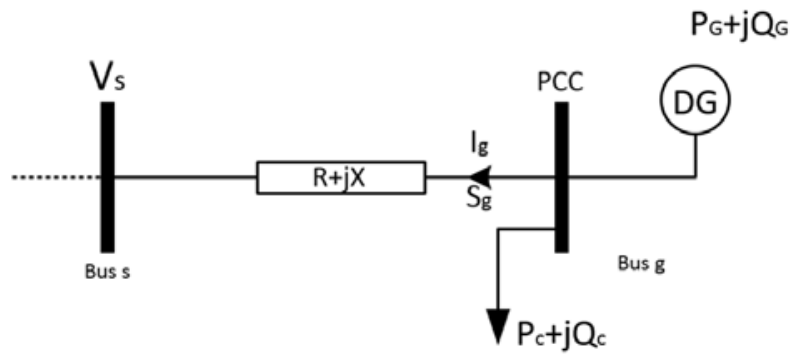

Figure 1: Two-Bus system with generator

$$
\Delta V_{s}=\frac{\Delta P_{g} R+\Delta Q_{g} X}{V_{g}}
$$

The main notion of the sensitivity analysis is to identify this amount of voltage variation regarding active and reactive power injections or absorptions.

\subsection{Classic theory of Sensitivity Analysis}

For transmission (mainly inductive) and some cases of MVDN (i.e. depending on $X / R$ ratio) it can be stated that the voltage variations are predominantly appeared due to reactive power flows. Underlying the latter which is also called decoupled approach is based on two observations [Greiger and Stevenson 1994]:

- Change in the voltage angle $\delta$ at a specific bus primarily affects the flow of real power $P$ in the transmission lines and leaves the flow of reactive power $Q$ relatively without change.

- Change in the voltage magnitude $|V|$ at a specific bus primarily affects the flow of reactive power $Q$ in the transmission lines and leaves the flow of real power $P$ relatively without change.

These observations lead to the typical Decoupled form of power flow equation. In that case the sensitivity factor can be calculated through the Jacobian matrix (2).

$$
\left[\begin{array}{l}
{[\Delta V]} \\
{[\Delta \vartheta]}
\end{array}\right]=\left[\begin{array}{ll}
{\left[\frac{\partial \boldsymbol{\Delta P}}{\partial V}\right]} & {\left[\frac{\partial \boldsymbol{\Delta P}}{\partial \vartheta}\right]} \\
{\left[\frac{\partial \boldsymbol{\Delta} Q}{\partial V}\right]} & {\left[\frac{\partial \Delta \boldsymbol{Q}}{\partial \vartheta}\right]}
\end{array}\right]^{-1} \cdot\left[\begin{array}{ll}
{[1]} & {[0]} \\
{[0]} & {[1]}
\end{array}\right] \cdot\left[\begin{array}{l}
{\left[\Delta P^{*}\right]} \\
{\left[\Delta Q^{*}\right]}
\end{array}\right]=[s] \cdot\left[\begin{array}{l}
{\left[\Delta P^{*}\right]} \\
{\left[\Delta Q^{*}\right]}
\end{array}\right]
$$


This consists the classical sensitivity which is sufficient to be used in transmission system analysis to perform primary and secondary voltage regulation [Brenna et al. 2013]. The [ $s$ ] matrix corresponds to the sensitivity indexes between nodal voltages (magnitude and phase angle) and active or reactive power injections. The computational complexity to perform the classic theory is augmented since the decoupling cannot be deployed due to the fact that the ratio $R / X$ varies.

\subsection{Alternative Sensitivity Approach}

In [Baran and Markabi 2007] the authors propose a distributed method of DG MAS dispatching to regulate nodal voltages. This method makes use of a valid approach of sensitivities with low computational complexity that can be estimated by each agent controller.

Assuming that in node $-i$ a (Figure 2 ) DG is connected which incrementally injects reactive power $\Delta Q$. The sensitivity factor $s_{j}$ depicts the impact of $\Delta Q$ injection downstream nodal voltages (i.e. $\Delta V_{i+1}$ ) and can be obtained easily obtained after some valid simplifications. The feeders of MVDN are purely radial in terms of operation, thus the voltage boost $\Delta V_{i}$ at node $i$ will be the same as all the downstream voltage variations, since the small change load changes can be neglected.

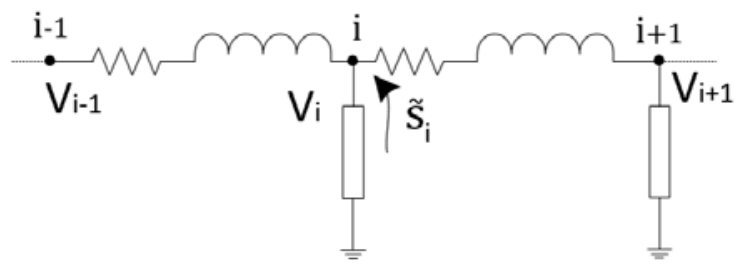

Figure 2: Part of a MVDN

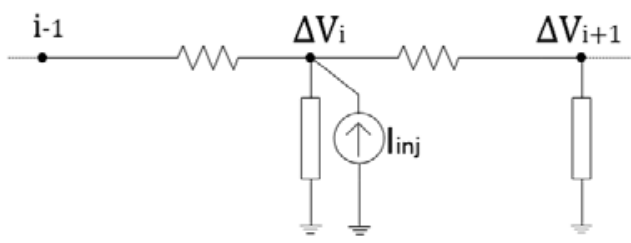

Figure 3: Equivalent DC-circuit

This observation originates from the fact that the linearized power flow equations correspond to a ladder dc network (Figure 3); hence the injection will provoke equal voltage raise to all nodal voltages. Therefore, the sensitivity factor of generator $i$ to downstream nodes can be evaluated from equation (3).

$$
\beta_{i}=\frac{\Delta V_{i}}{\Delta Q_{i}}
$$

This sensitivity method was simulated to the benchmark MVDN IEEE-13 nodes and verified that the assumptions were valid; while the accuracy of these sensitivity factors is particularly high. Nevertheless, the following approach concludes topological consideration to further simplify the calculation of all sensitivity terms within a consistent accuracy. In Figure 4 is observed that the voltage at each node tends to increment with the same tendency (i.e. parallel lines) after each reactive power injection occurred at node 8.

This sensitivity analysis provides a simplified alternative to identify the sensitivity factors regarding the downstream nodes. In 2.3 a more consistent approach of sensitivity analysis is presented based on topological considerations as well as radial DN traits. 


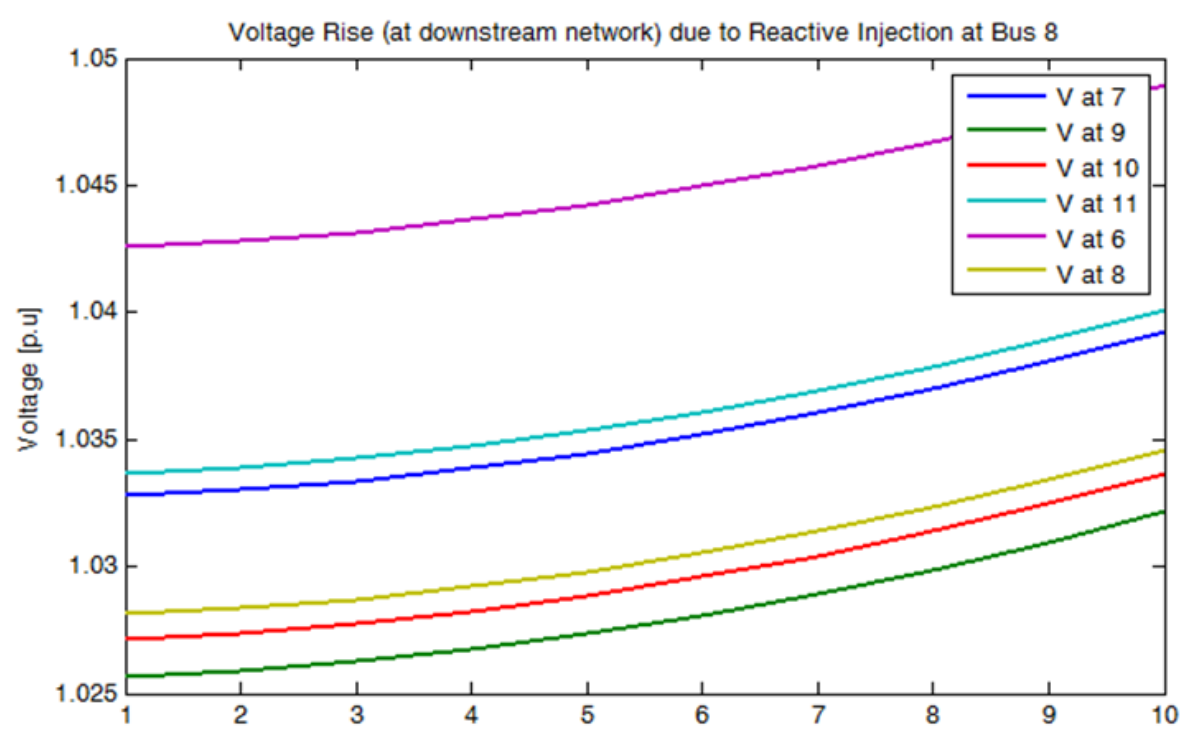

Figure 4: Nodal Voltages for each step of reactive injection at Node 8

\subsection{Sensitivity Analysis for Radial MDVN}

In [Brenna et al. 2013] a novel method to approach the sensitivity factors is given. Assuming a radial MVDN the quantification of voltage variations due to reactive power injections or absorptions of DG connected in bus $k$, can be methodologically estimated from (4).

$$
s_{Q_{i k}}=\frac{\partial V_{k}}{\partial Q_{i}}=\frac{1}{E_{n}} \cdot \sum_{\varphi \in \Pi} X_{\varphi}
$$

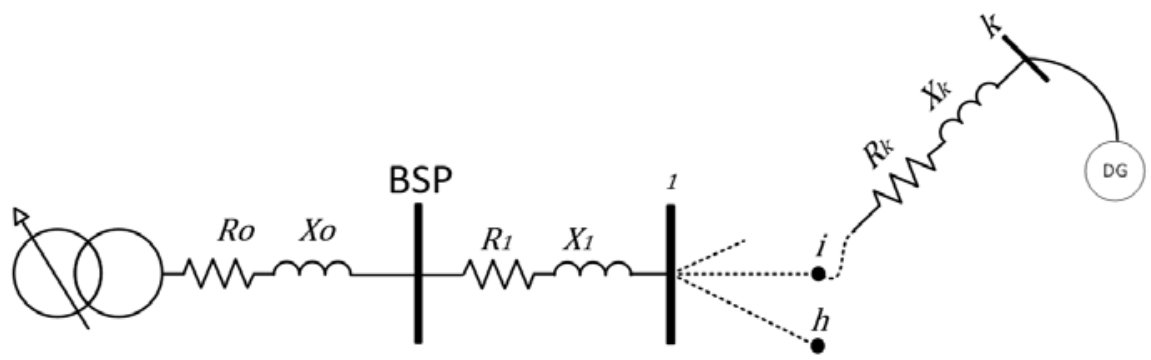

Figure 5: A per-phase equivalent circuit for a MVDN

Where in (4) $\Pi$ comprises the set that of the common path between node $k$ and the node of generator $-i$ from the Bulk Supply Point(BSP), $\Pi=\{$ Branches: $0,1 \ldots, i\}$. The term $E_{n}$ refers to the reference voltage of BSP and usually if there is no changes in the taps is set to 1 p.u. Respectively, the sensitivity terms for active variations can be defined in (5).

$$
s_{P_{i k}}=\frac{\partial V_{k}}{\partial P_{i}}=\frac{1}{E_{n}} \cdot \sum_{\varphi \in \Pi} R_{\varphi}
$$

This sensitivity analysis reveals that the impact on voltage raise or sag is inevitably connected with the topology of the network and the spatial correlation between the examined nodes. Therefore, for a given injection of $\Delta Q_{k}$ the final $i$ nodal voltage is given by (6) making use of sensitivity terms.

$$
E_{i}=\Delta E+E_{0}=s_{Q_{i k}} \cdot \Delta Q_{k}+E_{0}
$$

Based on this sensitivity theory a decentralized voltage control algorithm scheme is proposed in section 3. 


\section{Proposed Decentralized Voltage Regulation Scheme}

The proposed decentralized scheme is based on the exploitation of high DG penetration, and especially the efficient management of the available reactive power of each production unit for voltage regulation. The fact that multiple DGs are interconnected with the DN through oversized (i.e. technical requirement) smart inverters enables the likelihood to make use of their flexibility to actively participate in voltage regulation.

The key feature of the proposed is based on resolving voltage issues by the DGs that are adjacent or closest to the problematic node. The latter is inspired by the sensitivity analysis given in section 2.3 and contributes in the minimization of dispatched reactive power. Considering the topological specifications of radial networks, decisions are taken for the dispatch set-points of reactive power. This is in line with the new telecommunication technologies that have lead the way for a new peer-to peer (P2P) based model for the communication of the entities of the grid, where no central authority for control is needed and the structure is mostly self-organized, as well as the information is stored in a distributed way [Steinheimer et al. 2012]. This development provides a new insight in power system operation, where every entity shares equivalently the responsibility for the operation optimization communicating solely with its neighbors.

The above features are in absolute compliance with MAS based architectures, for this reason it is assumed that in all nodes of the network are installed agent controller that are capable to interact in between. Additionally, it assumed that nodes with DG interconnected, have DG agent controller which aggregates information regarding the current nodal voltage, Active power and reactive power point output as well as the sensitivity vector. The rest nodes have Load agent controller, whilst both agent types can interact requesting and accepting proposals for regulation support (Fig. 6). This figure is used for the explanation of the control strategy, depicting with red shadowed nodes that appeared to be out of the permissible bound while with green nodes where DGs are installed.

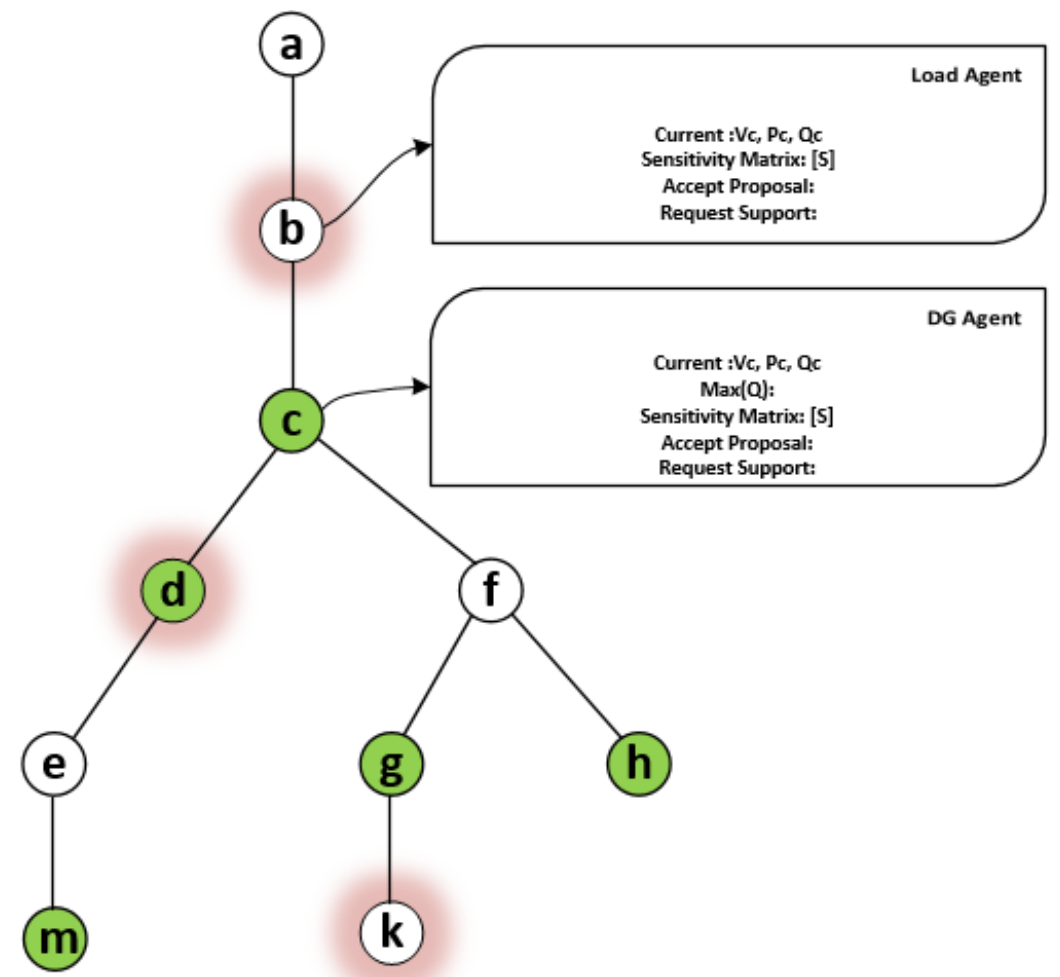

Figure 6: Graph depiction -part of network; green nodes represent those with DG; red shadowed with overvoltage or voltage sag issues 


\subsection{Portraying the proposed algorithm}

The proposed scheme is comprised by four different scalable levels as it is illustrated in Figure 7. The control algorithm is triggered by one or more nodes that appears voltage out of the permissible bounds. The first level refers to the instance that the problematic nodes compose DG node (i.e. installed generator), where the agent controller attempts to resolve the voltage issue by itself. More analytically, the agent using the reactive power sensitivity factor is feasible to estimate the anticipated amount of reactive power $(\Delta Q)$ which restore the voltage within limits.

$$
\Delta Q_{d}=\frac{\Delta E_{b}+E_{\text {hysteric }}}{s_{d d}}[p . u]
$$

On equation (7) $E_{\text {hysteric }}$ is set in 0,005 p.u to avoid reciprocating response of the control. The value was selected to be greater than the maximum error of all sensitivities (examined in section 4).

In case that more than one nodes have a non-permissible voltage along the same feeder then the upper node grants priority to the more distant (i.e. from the BSP) to commence voltage regulation. This is a tactic to minimize the reactive power variations, since any reactive power absorb contributes concurrently in a remarkable voltage boost to the adjacent upstream nodes.

The second level of the proposed scheme follows to perform voltage regulation. Apparently, levels 2-4 entail to resolve the voltage issue through a mixed integer linear optimization problem. The problematic node sends firstly requests for support to the direct connected nodes (e.g. adjacency); the query transmits if there is available DG unit that is willing to contribute in voltage regulation. For instance, (Fig. 6) agent will request support -within level 2 - from node $c$. Assuming that $c$ accepts to contribute; responds positive with the current maximum available reactive power. Following agent $g$ builds the optimization problem which aims to regulate the voltage of participating nodes with the minimum amount of reactive power variations. If that problem converges to a solution then the decided dispatch are sent to the contributors which are incorporate in set $A$. The formulation of the optimization problem is given by equations (8)-(9).

$$
\begin{gathered}
\min \boldsymbol{f}(Q)=\sum_{j \in A} \Delta Q_{j} \\
\text { s.t. }\left\{\begin{array}{c}
Q_{\min _{j}} \leq Q_{j}+\Delta Q_{j} \leq Q_{\text {max }_{j}} \\
V_{\text {min }} \leq V_{j}+\Delta V_{j} \leq V_{\text {max }}
\end{array}\right] \in A=\{\text { set of contributors }\}
\end{gathered}
$$

In case that there are no feasible solutions for the problem (8)-(9), the problematic node attempts request further support by enhancing the set of available DG agents $A$. This occurs at first requesting support from downstream connected DG units -within Level 3- since they present a reasonably greater sensitivity factor rather than the upstream. If the availability of contributing is low then the problematic node resorts to a further enhancement of set $A$ within level 4 of the proposed method. Regarding that level request are dispatched to upstream connected generators.

The decision variables of optimization problem are dispatched to the DG units; hence DGs vary their operating point injecting or absorbing the proper amount of reactive power.

The proposed method targets to regulate nodal voltages by using solely the available reactive power. This presupposes that the reactive power is managed in an efficient manner; following 
the proposed method selecting the most sensitive generator and prioritizing the most distant to resolve the voltage issue and finally the optimization problems, contribute significantly in minimizing the dispatched amount. Analytically the merit orders that are followed by the proposed scheme are illustrated in Fig. 7 in the format of a flow chart diagram.

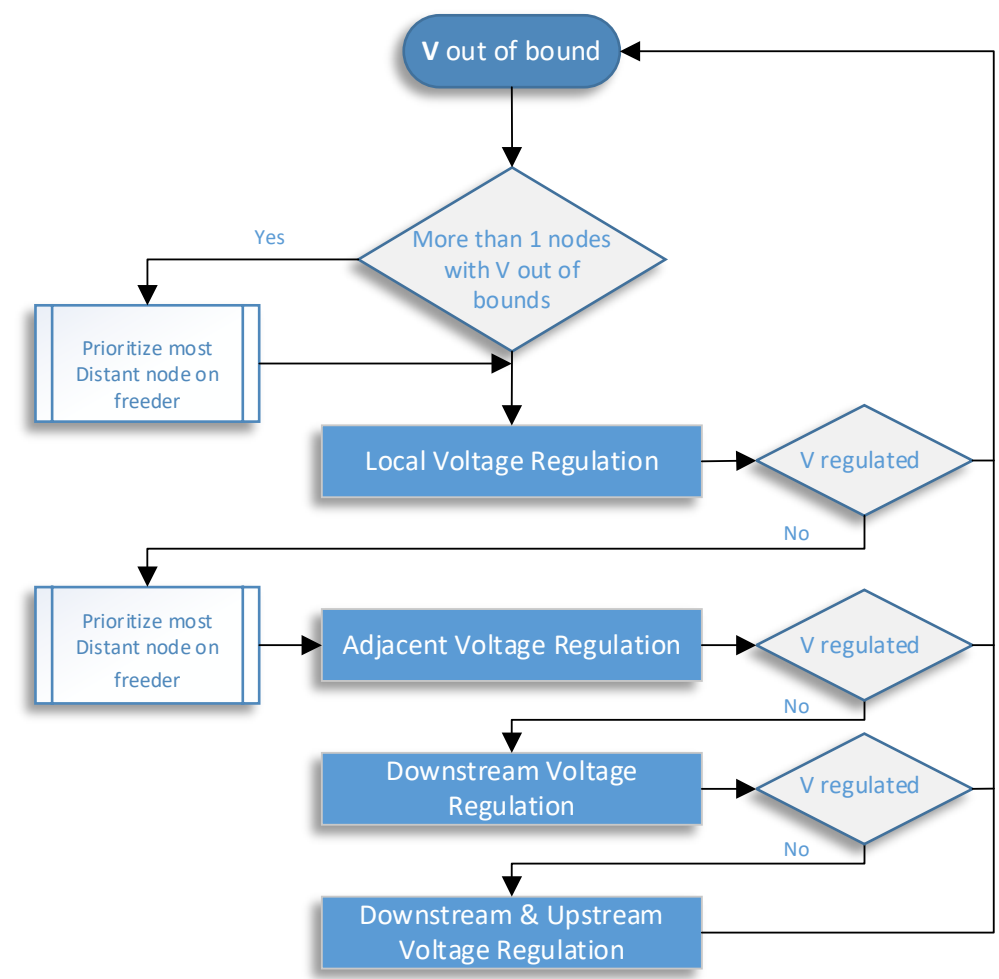

Figure 7: Simplified Flow of chart of proposed control strategy

\subsection{Verification of the proposed method}

This section targets on the validation of the proposed control strategy by performing simulations on MATLAB environment. The response of the decentralized method is compared with a centralized control scheme which theoretically converges to the optimal solution pertaining the minimal reactive power injections or absorption. The proposed method is tested for voltage sag instances where the DG units are challenged to inject reactive power thus to increment the nodal voltages. The centralized optimized (eq. 10) voltage requires the knowledge of all voltage measure of the network.

$$
\begin{gathered}
\min \boldsymbol{f}(Q)=\sum_{j \in G} \Delta Q_{j} \\
\text { s.t. }\left\{\begin{array}{c}
Q_{\text {min }_{j}} \leq Q_{j}+\Delta Q_{j} \leq Q_{\text {max }_{j}}, j \in G=\{\text { set of } D G s\} \\
V_{\text {min }} \leq V_{k}+\Delta V_{k} \leq V_{\text {max }}, k \in N=\{1, \ldots, n\}
\end{array}\right.
\end{gathered}
$$

The case study selected is derived from [Brenna 2011] while the characteristics of the loads and generators are selected from [Brenna 2013]. Some assumptions pertaining the simulated network:

- The substation transformer composes a BSP, capable to feed enough power to the loads in any case.

- Symmetric network (valid assumption in terms of European networks), which means symmetric loads and power sources.

- The loads are modelled ZIP model (constant complex power).

- The DG units initially operates in unitary power factor. 
For each simulated case the active power losses were examined although it is not an objective of the minimization problems. Nonetheless, the changes on DGs' power factor leads directly to a variation the reactive power flow between branches which in turn vary the nodal voltages. These have as consequence to impact on the active power losses which are of significant importance regarding the techno-economic operation of MVDN.

The installed capacity of each DG and the maximum value of reactive power (calculated with a minimum power factor) are illustrated in table 1.

\begin{tabular}{|c|c|c|}
\hline DG Unit & P[MW $]$ & $Q_{\max }[\mathrm{MVAr}]$ \\
\hline$D G_{1}$ & 5 & 2 \\
\hline$D G_{4}$ & 2.4 & 0.87 \\
\hline$D G_{6}$ & 10 & 3.63 \\
\hline$D G_{8}$ & 1.5 & 0.48 \\
\hline$D G_{10}$ & 1 & 0.73 \\
\hline$D G_{11}$ & 3.5 & 1.49 \\
\hline
\end{tabular}

Table 1: Nominal Capacity of installed DG units

As first the accuracy of sensitivity factors is performed, in order to ensure the consistency of the method. The sensitivity factors were used to estimate the nodal voltage after injections of reactive power from the DG units. In Fig. 10a and $10 \mathrm{~b}$ is illustrated analytically the error per each estimation. It is determined that the error due to the excess of linearization (i.e. significant dispatched power) leads a rational incrementation of error. It is plausible to stress that in case of two simultaneous dispatches the error is augmented. Nevertheless, the error can be avoided by using the hysteric in the term of estimation as it was shown in the previous section. Additionally, for consistency the permissible bounds of voltage are set in the vicinity of $[0.97,1.03]$.

For a particular case of operating point where the load is $50 \%$ of the nominal installed and the generation of DG is on $100 \%, 5$ nodal overvoltages (nodes coloured in red $6,7,8,10,11$ ) are detected from the agents which intend to resolve the problem following the proposed tactics. In Fig. 8 is depicted the graph of the network for this operating point, where the arrows symbolize the power flows among the branches.

Following the proposed strategy, the first level is to resolve the voltage problem from the problematic nodes that contain DG node given the prioritization. The DG agent 8 is informed from the downstream voltage issue, who gives priority to the most distant node 10 (that is examined through the vector of sensitivity). In this step the DG10 is available to absorb 0.325MVAr firstly restoring its voltage contributing with a voltage drop as it is illustrated in Fig. 9a to all nodes. Afterwards, DG agent 8 estimates that can locally resolve the overvoltage absorbing $0.667 \mathrm{MVAr}$. The nodes $6,7,11$ are still out of the permissible bound; hence the DG agent 6 is the node that commences the procedure of requesting support. Since node 6 composes a leaf, proceeds in sending requests for support to the upstream network, where DG 8 responds to this request by sending his maximum reactive availability. In agent 8 , the optimization problem with set $A=\{6,8\}$, which converges to a solution with set of variables $[x]=\left[\Delta Q_{6} \Delta Q_{8}\right]^{T}=[0.370 .116]^{T} p . u$.

The overall reactive variations decided through the proposed control strategy is 5.748MVAr. 


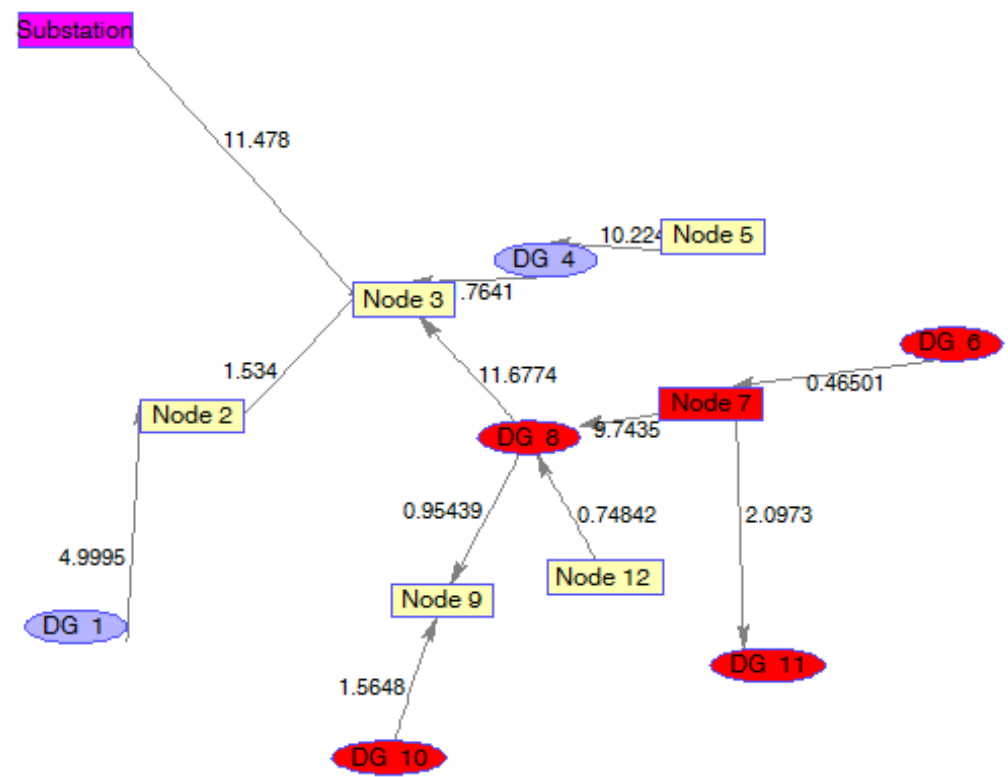

Figure 8: Graph of 13 Test Node network (Load 50\% - Generation 100\%)

The respective centralized optimization problem which considers full network model knowledge and the nodal voltages decides to dispatch 5.449 MVAr in order to restore voltages. The active power losses of the proposed method are $0.321 \mathrm{MW}$ while in the central method $0.29 \mathrm{MW}$. This difference on active power losses is mainly due to different feeder voltage profile occurred after each case of decision made regarding the dispatch.

a)

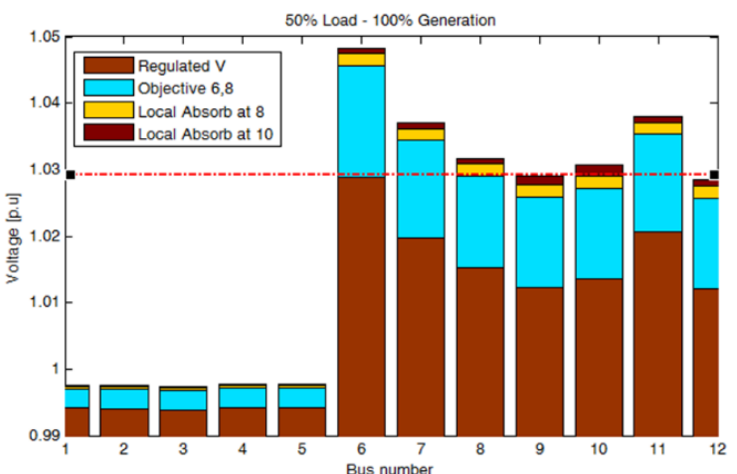

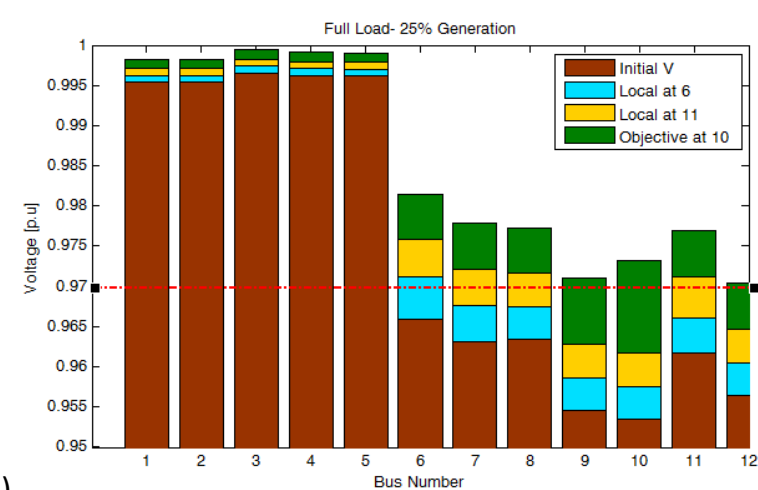

b)

))
Figure 9: The voltage regulation is illustrated in steps of the decisions a) Graph of $100 \%$ DG Generation and 50\% nominal load- overvoltage in 5 nodes b) 25\% DG Generation and full loadundervoltage in 7 nodes

Assuming an instance when the generated power from the DG is $25 \%$ of the installed power and the load is on its peak. For that case there are 7 nodes which appear undervoltage. As it is illustrated in Fig. 9b the DG agents 6 and 11 proceed to local voltage regulation by injecting 1,409MVAr and 1,468MVAr respectively. The overall voltage issue is resolved by agent 10 which constructs optimization problem with DG 8. The total dispatched power is 5.805MVAr; whilst the central technique decides to dispatch 5.8 MVAr.

The initial active power losses on this operating point were $0.617 \mathrm{MW}$; after the regulation through the proposed method are $0.897 \mathrm{MW}$ while the central method gives $0.883 \mathrm{MW}$. It is important to be stressed that the minimization of active power losses is not herewith an objective, but a techno-economic parameter that must be monitored. 
a)

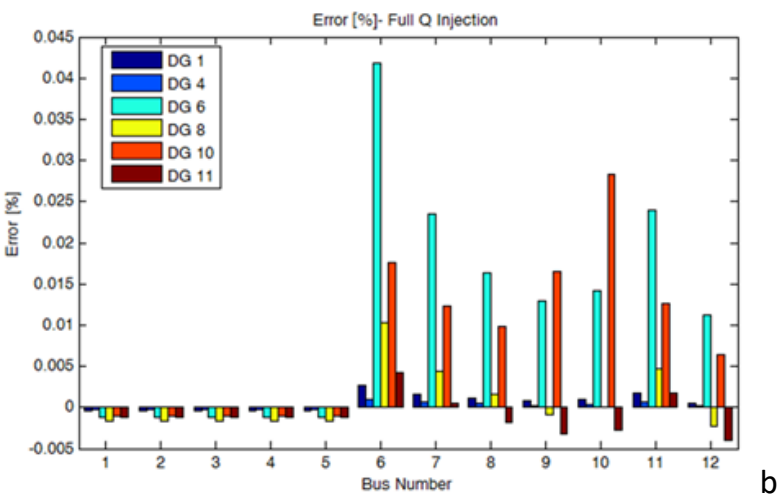

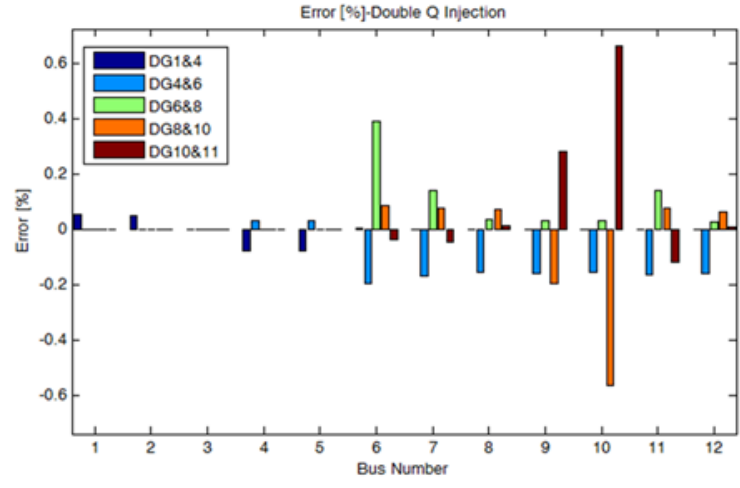

b)

Figure 10: The error estimation by using sensitivity factors a) Dispatch one DG each time b) Dispatch two DGs

The simulated cases appear to present decisions very close to the central dispatch scheme which converges to the minimum appropriate reactive power contribution to regulate voltages. The proposed algorithm was simulated in topologies with 36 and 50 nodes, where the topologies have longer feeders and the necessity to resolve the voltage issue locally is crucial. Through the simulation it is also identified that the prioritization to the most distant nodes contributes significantly to the reduction of the dispatched reactive power. In Fig. 10 it is presented the simulation made to identify the maximum sensitivity that namely occur due to linearization approach. Although the maximum error is addressed by introducing lower voltage limits and a hysteric limit on the proposed technique.

\section{Conclusions}

The continuous growth of integration of dispersed generation into the MVDN introduces multifaceted technical challenges that can be addressed by the transition to the Active Network Management. The active participation of DGs into the control and regulation schemes can be accomplished by controlling their active or reactive power. An active power curtailment implies decreased income for the agent generator. The proposed decentralized control strategy targets on the nodal voltages by the managing the power factor of the most sensitive -to the problematic node- DG. This substantially leads to an efficient dispatch of DG resolving the voltage issues.

In this work different sensitivity theories approaches are aggregated and examined regarding their methodology and complexity of calculation as well as their accuracy. The sensitivity factors for active and reactive power variations can be finally approached by the topological structure of network while there is no intensive influence from the different loading states of the network.

The proposed control schemes resolves the voltage issues in both cases of overvoltages or voltage sags in multiple nodes, by absorbing or injecting respectively the proper amount of reactive power. Provided the comparison with the centralized scheme the proposed methodology converges in the optimal solution of the minimum reactive power dispatches. This is occurred since any voltage issue is resolved locally which directly implies the dispatch of the most sensitive DG units. At the same time the feature of prioritization of excessive voltage nodes on each feeder, leads to a further decrease of total reactive variations.

\section{Future Work}

As it was discussed in the introductory section the need to transit to more intelligent and smart control techniques. The proposed control is hereby validated in a simulation environment, although it would be interesting to be implemented on a real field application 
using a MAS based on P2P communication protocol. On this work the main focus was on the control strategy, nevertheless it is of great importance to investigate the proper time settings for the communications as well as the triggering of the control.

Additionally, an interesting approach would be to embed this control strategy in a coordinated scheme with the contribution of conventional means which were aforementioned in the introduction.

Another possible further development would be the extend of this control strategy on Low Voltage DN, which appeared to be purely unbalanced. This would require a further adjustment on the approach of sensitivities. Additionally, on this case of LVDN an interesting contribution possibly be the control of active power output of DGs since the LV networks are namely resistive.

\section{References}

H. Reponen, A. Kulmala, V. Tuominen and S. Repo. 2016. "RTDS Simulation of Coordinated Voltage Control in Low Voltage Distribution Network". Proceedings in IEEE ISGT 2016, Slovenia.

J.A. Pecas Lopes, N. Hatziargyriou, J. Mutale, P. Djapic and N. Jekins. 2007. "Integrating distributed generation into electric power systems: A review of drivers, challenges and opportunities", Electric Power Systems Research 77 (2007) 1189-1203, ELSEVIER.

H. E. Farag, E. El-Saadany, R. Seethapathy. 2012."A Two Ways Communication-Based Distributed Control for Voltage Regulation in Smart Distribution Feeders", in IEEE Transactions on Smart Grid, Vol. 3 N0. 1, pp. 271-281.

P. N. Vovos, A. E. Kiprakis, A. R. Wallace, G. P. Harrision. 2007. “Centralized and Distributed Voltage Control: Impact on Distributed Generation Penetration", in IEEE Transactions on Power Systems, Vol. 22, NO. 1, pp. 476-483.

Y. Rosales Hernandez and T. Hiyama. 2008. "Distance measure based rules for voltage regulation with loss reduction," in Proceedings IEEE Power Energy Soc. Gen. Meet.Convers. Del. Electr. Energy 21st Century.

F. Saccomanno. 2003. "Electric Power Systems". Interscience IEEE Press, Piscataway, NJ: Wiley.

P. H. Nguyen, W. L. Kling, and J. M. A. Myrzik, , 2007. "Promising concepts and technologies for future power delivery systems", UPEC, pp.47-52.

Z. Jiang. 2006. "Agent-based control framework for distributed energy resources microgrids," in Proc. IEEE/WIC/ACM Int. Conference Intelligent Agent Technology, pp. 646-652.

P. Lund. 2007. "The danish cell project-part 1: Background and general approach," in Proc. IEEE Power Eng. Soc. Gen. Meet., Tampa, FL, pp. 1-6.

J. Oyarzabal, J. Jimeno, J. Ruela, A. Engler, and C. Hardt. 2005. “Agent-based-micro-grid management- system," in Proc. Int. Conf.Future Power Syst., pp. 1-6.

M. Pipattanasomporn, H. Feroze, and S. Rahman. 2009."Multi-agent systems in a distributed smart grid: Design and implementation," in Proc.IEEE Power Syst. Conf. Expo, Seattle, WA, pp. 1-6.

P. H. Nguyen, J. M. A. Myrzik, and W. L. Kling. May 2008. "Coordination of voltage regulation in active networks," in roc. Transm. Distrib. Conf. Expo., Chicago, IL, pp. 1-6.

J. J. Greiger, W. D. Stevenson. 1994. "Power System Analysis”, Mc-Graw Hill. 
M. E. Baran, I. El-Markabi. 2007. “A Multiagent-Based Dispatching Scheme for Distributed Generators for Voltage Support on Distribution Feeders", in IEEE Transactions on Power Systems, Vol. 22, NO. 1, pp. 52-59.

Steinheimer, M. Trick, U., Ruhrig, P., 2012"Energy communities in Smart Markets for optimization of peer-to-peer interconnected Smart Homes", Communication Systems, Networks \& Digital Signal Processing (CSNDSP), 8th International Symposium on, vol., no., pp.1,6, 18-20.

M. Brenna, E. De Berardinnis, L. D. Carpini et al., 2013. "Automatic Distributed Voltage Control Algorithm in Smart Grid Applications", in IEEE Transactions on Smart Grid, Vol. 4, NO. 2, pp. 877-885.

M. Brenna, F. Foiadelli, and D. Zaninelli. 2011. "New stability analysis for tuning PI controller of power converters in railway application," IEEE Transaction Industrial. Electron., vol. 58, no. 2 , pp. 533-543. 